\title{
Purification, characterization and procoagulant activity of polysaccharides from Angelica dahurice roots
}

Jinmei Wang ${ }^{1 \dagger}$, Pengli Lian ${ }^{1,2+}$, Qi Yu ${ }^{1}$, Jinfeng Wei ${ }^{1,2^{*}}$ and Wen-yi Kang ${ }^{1,2^{*}}$

\begin{abstract}
Five polysaccharides, namely ADPs-1a, ADPs-1 b, ADPs-2, ADPs-3a and ADPs-3b, were extracted from Angelicae dahuricae Radix, purified, and identified by high performance gel permeation chromatography (HPSEC), gas chromatography (GC), Fourier transform infrared (FT-IR) spectrometer and nuclear magnetic resonance spectra (NMR), including the determination of procoagulant activity in vitro. The average molecular weight $(\mathrm{MW})$ of the polysaccharides was 153,800, 8312, 111,700, 3766 and 96,680 g/mol, respectively. Coagulation assays indicated that ADPs-1b, ADPs-2, ADPs-3a and ADPs-3b had procoagulant activities. ADPs-1 $b$ exerted the procoagulant activities through intrinsic pathway, extrinsic pathway and increased the content of FIB in vitro. ADPs-2 exerted the procoagulant activities through intrinsic pathway and extrinsic pathway. ADPs-3a had procoagulant activities and the activity was associated with the intrinsic pathway and increased the content of FIB. ADPs-3b exerted the activities through extrinsic pathway and increased the content of FIB.
\end{abstract}

Keywords: Angelicae dahuricae Radix, Polysaccharides, Procoagulant

\section{Background}

Angelicae dahuricae Radix, named 'Baizhi' in Chinese, has been a well-known traditional dietary and medicinal plant for several 1000 years. It has traditionally been used for treatment of headache caused by the common cold, asthma, coryza, hypertension, vitiligo, psoriasis and photodynamic therapy. Pharmacological research showed that A. dahuricae Radix had antioxidant [1], antibacterial [2], anti-tumor [3] and analgesic [4] activities. The bioactive components mainly contained coumarins [5], volatile oils [6], polysaccharides [7] and trace elements [8]. Coumarins had been intensively studied in the literatures, and polysaccharides were rarely studied. However. Polysaccharides have many biological activities. It has been reported that polysaccharides from A. dahuricae Radix

\footnotetext{
*Correspondence: weijinfeng20112011@hotmail.com;

kangweny@hotmail.com

${ }^{\dagger}$ Jinmei Wang and Pengli Lian contributed equally to this work

${ }^{1}$ Institute of Chinese Materia Medica, Henan University,

Kaifeng 475004, China

Full list of author information is available at the end of the article
}

had antioxidant activity [9], can promote the proliferation of rat skin cells cultures in vitro [10] and enhance the ability of F81 cells to resist canine parvovirus infection [11].

The literature search showed the water extracts from $A$. dahuricae Radix had obvious hemostatic effect [12]. Till date there is no investigation reported on the bioactive components of water extract from A. dahuricae Radix for hemostatic effects. In this paper, water-soluble polysaccharides were extracted and purified, and its procoagulant activity in vitro was studied which could provide a evidence for clinical application of polysaccharides from A. dahuricae Radix.

\section{Methods}

\section{Plant material}

Angelicae dahuricae Radix were purchased in April 2013 from the golden pieces of Chinese Medicine Co., Ltd. of Yuzhou (Henan, China) and were identified by Prof. Chang-qin Li. The voucher specimens were deposited at traditional Chinese medicine research Institute of Henan University. 


\section{Animals}

Male rabbit (2.0-2.5 kg), was obtained from the Experimental Animal Center of Henan Province (Zhengzhou, Henan, China, No: 14-3-7). It was maintained under a $12 / 12 \mathrm{~h}$ light/dark cycle, at $25 \pm 2{ }^{\circ} \mathrm{C}$ and humidity 45-65\%, with free access of food and water. The animal procedures were approved by the ethical committee in accordance with 'Institute ethical committee guidelines' for Animal Experimentation and Care. Animals were housed in standard cage.

\section{Reagents}

DEAE-cellulose-52 (Whatman, Germany); Sephadex G-100 (Pharmacia, America); TGL-16 high speed centrifuge (Zhongda instrument factory, Jintan, China); HF6000 Semi-Automated Coagulation Analyzer (Chinese Prescription Medical Instrument Co., Ltd, Jinan, China); LRH-150 incubator (Shanghai Yiheng Technology Co. Ltd., China); stopwatch timer; vitamin $k_{1}$ injection, $2.775 \mathrm{~g} / \mathrm{L}$ calcium chloride solution, (Tianjin Pharmaceutical Group Co., Ltd. Xinzheng, 1109051); APTT (Lot: 112163), PT (Lot: 105227), TT (Lot: 121116), FIB (Lot: 132058) assay kits (Shanghai sun biotech Co., Ltd.).

\section{Extraction and purification of polysaccharide}

Angelicae dahuricae Radix (100 g) were grounded into powder, then extracted three times with $70 \%$ ethanol and filtered. Subsequently, the dried power was dipped into 20 volumes of distilled water at $80{ }^{\circ} \mathrm{C}$ every $3 \mathrm{~h}$ for three times. The aqueous extract was filtered and the supernatant was treated with $95 \%$ ethanol (final concentration $70 \%$ ) at $4{ }^{\circ} \mathrm{C}$ overnight, and centrifuged at $10,000 \mathrm{rpm}$ for $10 \mathrm{~min}$. The precipitation was added with Sevage reagent (chloroform/1-butanol, 1:4 v/v) for deproteinization. The crude polysaccharide was obtained through precipitation with 95\% ethanol (final concentration 70\%) and centrifuged. Then the precipitation was redissolved in water and dialyzed against distilled water for 2 days. Finally, the aqueous extract was lyophilized in vacuum to give the crude polysaccharide $(5.11 \mathrm{~g})$.

The crude polysaccharides $300 \mathrm{mg}$ was dissolved in $10 \mathrm{~mL}$ distilled water, filtered through $0.45 \mu \mathrm{m}$ filters and then fractioned by DEAE- 52 column $(2.5 \times 60 \mathrm{~cm})$. The column was eluted with distilled water at $0.8 \mathrm{~mL} /$ min, followed by $0.05 \mathrm{M} \mathrm{NaCl}$ and $0.1 \mathrm{M} \mathrm{NaCl}$, respectively. The fractions were collected using an automated step-by-step fraction collector and guided for total carbohydrate using the phenol-sulfuric acid method. Three main fractions were collected, dialyzed, lyophilized. These polysaccharides were further purified through a column of Sephadex G-100 $(1.5 \times 100 \mathrm{~cm})$ and eluted with water at $0.5 \mathrm{~mL} / \mathrm{min}$. The purified fraction was combined, concentrated and lyophilized for further study.

\section{Structural analysis Molecular weight analysis}

The molecular weight of polysaccharides was identified by high performance size-exclusion chromatography (HPSEC) in Beijing center for physical and chemical analysis.

\section{Monosaccharide composition analysis}

Polysaccharide samples (10 mg) were hydrolyzed in ampoules with $2 \mathrm{M}$ trifluoroacetic acid $(2 \mathrm{~mL})$ for $3 \mathrm{~h}$ at $110{ }^{\circ} \mathrm{C}$, evaporated and added with methanol to remove TFA. Then the hydrolyzates were mixed with $10 \mathrm{mg}$ hydroxylamine hydrochloride and $0.5 \mathrm{~mL}$ pyridine and incubated at $90{ }^{\circ} \mathrm{C}$ for $30 \mathrm{~min}$. Acetic anhydride $(0.5 \mathrm{~mL})$ was added and incubated at $90{ }^{\circ} \mathrm{C}$ for $30 \mathrm{~min}$. The mixtures were cooled to room temperature, and filtered through $0.22 \mu \mathrm{m}$ filters. The resulting alditol acetates were analyzed by GC, which was performed on a Thermo TRACE1300 instrument fitted with FID $\left(280{ }^{\circ} \mathrm{C}\right)$ and equipped with HP-5 column $(30 \mathrm{~m} \times 0.25 \mathrm{~mm} \times 0.25 \mu \mathrm{m})$. The column temperature was maintained at $110{ }^{\circ} \mathrm{C}$ for $5 \mathrm{~min}$, and increased to $190{ }^{\circ} \mathrm{C}$ for $4 \mathrm{~min}$ at a rate of $5^{\circ} \mathrm{C} / \mathrm{min}$, then increased to $210{ }^{\circ} \mathrm{C}$ for $10 \mathrm{~min}$ at a rate of $3{ }^{\circ} \mathrm{C} / \mathrm{min}$. The standard monosaccharides (arabinose, xylose, mannose, glucose and galactose) were prepared and subjected to GC analysis separately in the same way.

\section{FT-IR spectral analysis}

Polysaccharides were grounded with $\mathrm{KBr}$ power, pressed into pellets and then detected in the frequency range of $4000-50 / \mathrm{cm}$.

\section{NMR spectral analysis}

The NMR spectra of ADP-1a, ADP-1b, ADP-2, ADP$3 \mathrm{a}$, and ADP-3b were obtained by an Avance-600 NMR spectrometer (Bruker Inc., Rheinstetten, Germany). All compounds were dissolved in $\mathrm{D}_{2} \mathrm{O}$. The ${ }^{1} \mathrm{H}$ NMR spectra of ADP-1a, ADP-1b, ADP-2, ADP-3a, and ADP-3b were recorded, ${ }^{13} \mathrm{C}$ NMR spectra, the $2 \mathrm{D}$ NMR spectra including heteronuclear multiple-quantum coherence (HMQC) and heteronuclear multiple bond correlation (HMBC) of ADP-1a and ADP-2 were recorded.

\section{Scanning electron microscope analysis}

Polysaccharide samples were fixed on the sample stage, subsequently coated with a layer of gold, and then scanned by scanning electron microscope. 


\section{Anticoagulation time test}

Anticoagulation activities of APTT, PT, TT and FIB were analyzed in vitro and the assay was conducted by using rabbit blood collected from rabbit ear vein in the plastic tubes containing $3.8 \%$ sodium citrate (citrate/blood: $1 / 9$, $\mathrm{v} / \mathrm{v}$ ). Then, the blood was centrifuged at $3000 \mathrm{rpm}$ for $15 \mathrm{~min}$ at $5{ }^{\circ} \mathrm{C}$ to obtain the serums. For APTT assay, 25 $\mu \mathrm{L}$ of tested samples were mixed with $50 \mu \mathrm{L}$ of citrated normal rabbit serum, and then APTT assay reagent was added. Following the mixture were incubated at $37^{\circ} \mathrm{C}$ for $5 \mathrm{~min}$. Then $25 \mathrm{mM} \mathrm{CaCl}$ solution $(100 \mu \mathrm{L})$ was added into the incubated mixture to initiate the reaction. Finally the clotting time was recorded. For PT assay, samples $(25 \mu \mathrm{L})$ were mixed with serum $(25 \mu \mathrm{L})$ and incubated at $37{ }^{\circ} \mathrm{C}$ for $3 \mathrm{~min}$. While, PT assay reagent $(50 \mu \mathrm{L})$, which has been hatched for $10 \mathrm{~min}$ at $37^{\circ} \mathrm{C}$, was then added and clotting time was recorded. TT and FIB assays were performed according to the manufacture's specifications. For all clotting assays, blank solvent was used as blank control group, and breviscapine and Vitamin $K_{1}$ were used as positive control group and the time for clot formation was recorded by Semi-Automated Coagulation Analyzer.

\section{Statistical analysis}

All experimental results were expressed as mean \pm standard deviation (SD). Statistical analysis was performed with the SPSS 19.0 software. Comparison between any two groups was evaluated using one-way analysis of variance (ANOVA).

\section{Results and discussion}

\section{Extraction and purification of polysaccharide}

Crude polysaccharides $(300 \mathrm{mg}$ ) were successfully isolated by a series of experimental procedures such as water extraction, deproteination, dialysis, ethanol precipitation and lyophilization. The crude polysaccharides were then separated by using DEAE-cellulose-52 column. Three purified polysaccharide fractions were obtained, named ADP-1 (91.2 mg), ADP-2 (36.5 mg) and ADP-3 (73.6 mg) (Fig. 1a), respectively. Three fractions were further purified by Sephadex G-100. As a result, ADP-1 generated two purified fraction, named as ADPs1a (30 mg) and ADPs-1b (32.4 mg) (Fig. 1b). ADP-2 generated one purified fraction, named as ADPs-2 $(28 \mathrm{mg})$ (Fig. 1c). ADP-3 generated two purified fraction, named as ADPs-3a (36.2 mg) (Fig. 1d) and ADPs-3b (21 mg) (Fig. 1d).

\section{Molecular weight analysis}

Molecular weight of polysaccharide was a statistical average, which was a representative of similar polymer chain length distributed on average. Generally,

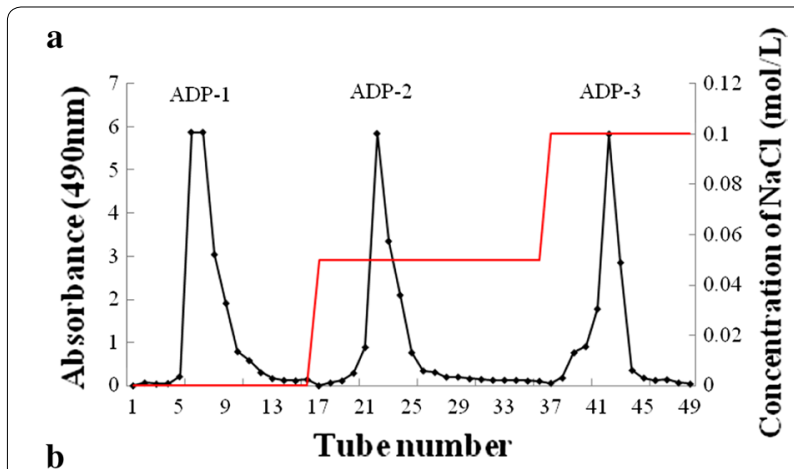

b

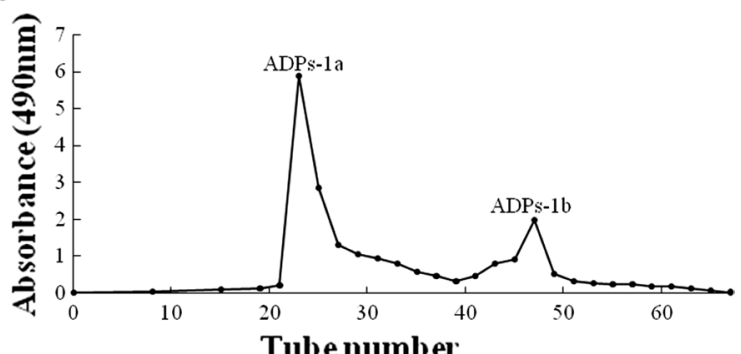

c

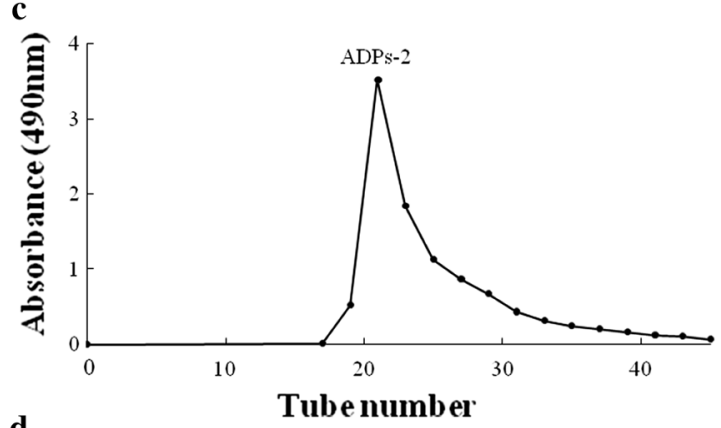

d

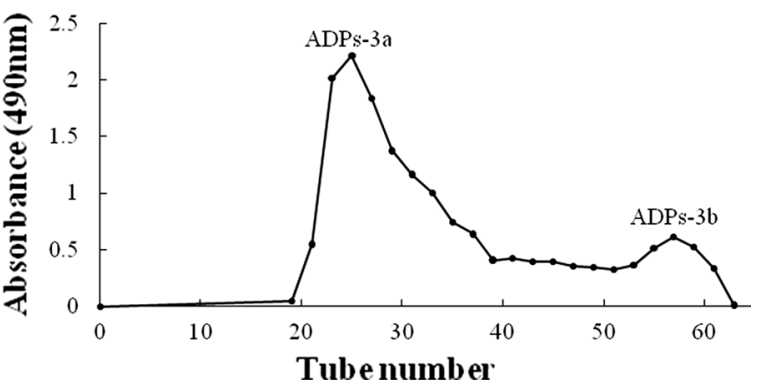

Fig. 1 Elution curve of the crude polysaccharides on DEAE-52 (a), elution curve of ADP-1 on Sephadex G-100 column (b), elution curve of ADP-2 on Sephadex G-100 column (c), elution curve of ADP-3 on Sephadex G-100 column (d)

the dispersion coefficient $(M w / M n)$ was used to be a judgment whether the molecular weight distributed uniformly or not. As it is shown in Table 1, average molecular weight $(\mathrm{Mw})$ of the polysaccharides was $1.538 \times 10^{5}, 8.312 \times 10^{3}, 1.117 \times 10^{5}, 3.766 \times 10^{3}$ and $9.668 \times 10^{4} \mathrm{~g} / \mathrm{mol}$, respectively. 
Table 1 Molecular weight of polysaccharides form Angelicae dahuricae Radix

\begin{tabular}{|c|c|c|c|c|c|}
\hline \multirow[t]{2}{*}{ Samples } & \multicolumn{5}{|c|}{ Molecular weight $(\mathrm{g} / \mathrm{mol})$} \\
\hline & $M n$ & $M p$ & $M w$ & $M z$ & $M w / M n$ \\
\hline ADPs-1a & $1.03 \times 10^{5}$ & $9.541 \times 10^{4}$ & $1.538 \times 10^{5}$ & $2.896 \times 10^{5}$ & 1.493 \\
\hline ADPs- $1 b$ & $7.942 \times 10^{3}$ & $8.187 \times 10^{3}$ & $8.312 \times 10^{3}$ & $8.872 \times 10^{3}$ & 1.047 \\
\hline ADPs-2 & $8.076 \times 10^{4}$ & $7.794 \times 10^{4}$ & $1.117 \times 10^{5}$ & $1.832 \times 10^{5}$ & 1.384 \\
\hline ADPs-3a & $3.518 \times 10^{3}$ & $3.551 \times 10^{3}$ & $3.766 \times 10^{3}$ & $4.327 \times 10^{3}$ & 1.070 \\
\hline ADPs-3b & $6.751 \times 10^{4}$ & $8.017 \times 10^{4}$ & $9.668 \times 10^{4}$ & $1.815 \times 10^{5}$ & 1.432 \\
\hline
\end{tabular}

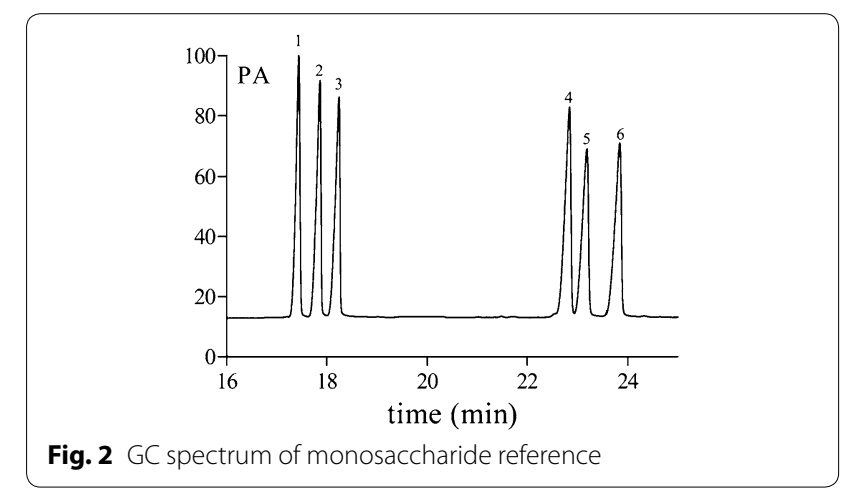

\section{GC analysis}

Monosaccharide composition was analyzed by gas chromatography. Based on retention times and content based on every monosaccharide by authentic standards (Figs. 2, 3), the monosaccharide composition of ADPs-1a was xylose, mannose, glucose and galactose in a molar ratio of 0.31:0.22:26.1:0.11. ADPs-1b was composed of arabinose, xylose, mannose, glucose and galactose with a molar ratio of $0.10: 0.26: 0.07: 15.3: 1.37$. The monosaccharide compositions of ADPs-2, ADPs-3a and ADPs-3b were rhamnose, arabinose, xylose, mannose, glucose and galactose. ADPs- 2 was in a ratio of 0.34:1.79:0.35:0.40:15.8:5.59. ADPs-3a was in a ratio of 1.06:2.01:0.13:0.41:1.68:4.97 and ADPs-3b was in a ratio of $0.18: 0.36: 0.25: 0.09: 13.5: 1.59$. According to the literature [13], monosaccharide constituents of ADP were rhamnose, arabinose, xylose, mannose, glucose and galactose, thus differed from our reports and these difference might be related to the source of the $A$. dahuricae Radix, extraction and purification methods.
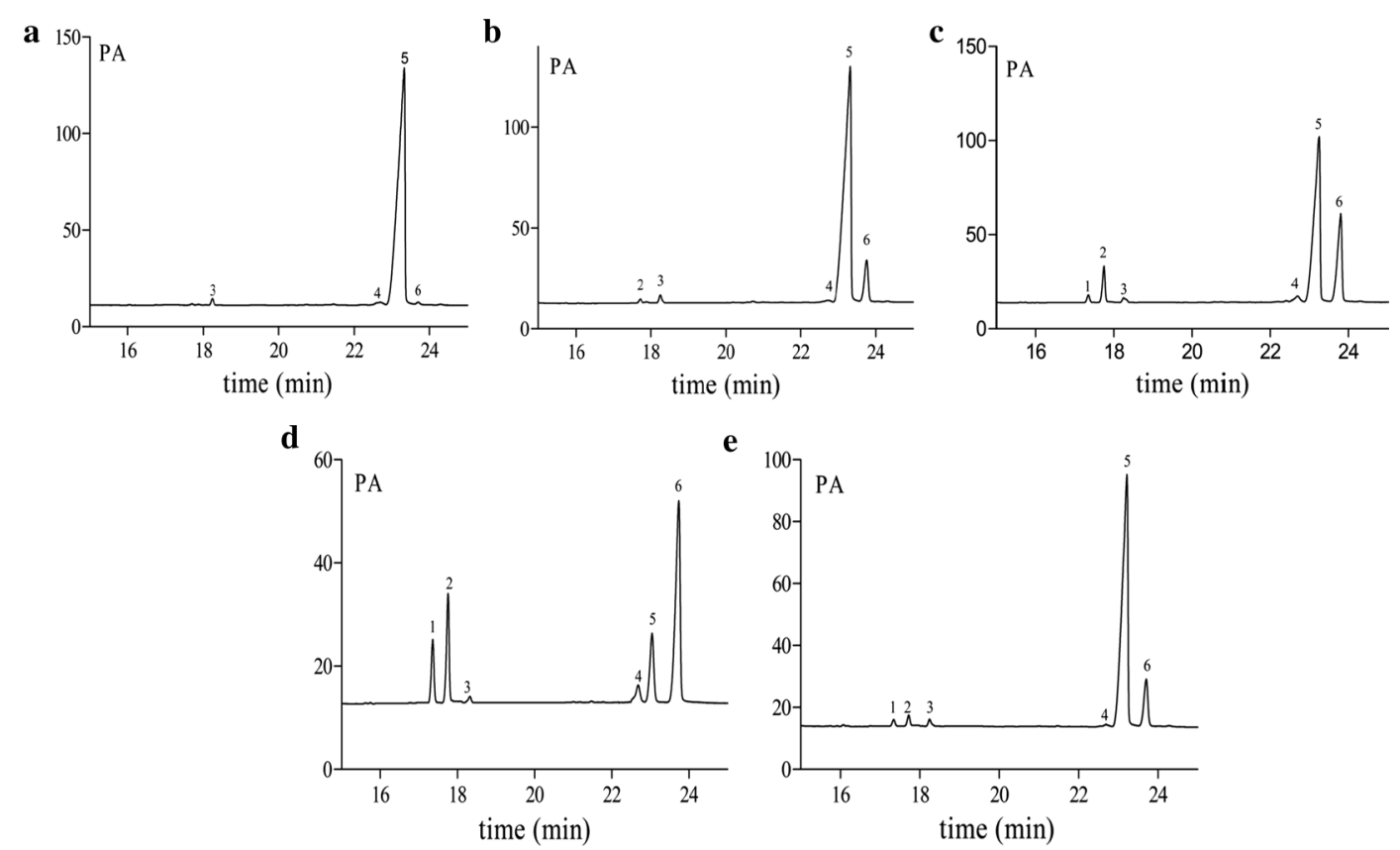

Fig. 3 GC spectrum of monosaccharide composition of ADPs-1a (a), ADPs-1b (b), ADPs-2 (c), ADPs-3a (d) and ADPs-3b (e) 


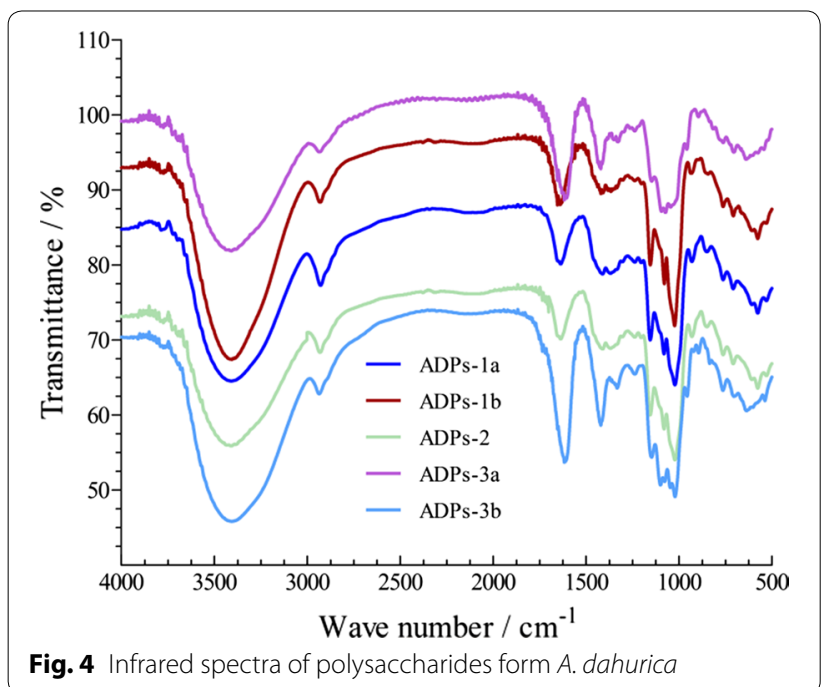

\section{Ft-ir}

The FT-IR spectroscopy of ADPs-1a, ADPs-1b, ADPs-2, ADPs-3a and ADPs-3b were scanned between 4000 and $500 / \mathrm{cm}$ and the results showed the four polysaccharides were similar to each other. As shown in Fig. 4, the absorption band was found in all samples between 3321 and $3375 / \mathrm{cm}$, indicating the presence of hydroxyl group. The appearance of the peaks within the range of 2772-2922/ $\mathrm{cm}$ was due to the presence of the $\mathrm{C}-\mathrm{H}$ stretching vibration. The signals at around $1592-1625 / \mathrm{cm}$ and $1424-$ 1440 were showing the presence of carboxyl groups. Absorption at $1010-1125$ the $\mathrm{C}-\mathrm{O}$ and $\mathrm{C}-\mathrm{C}$ stretching vibrations of pyranose ring.

\section{NMR spectral analysis}

As shown in Fig. 5, the anomeric region of the ${ }^{1} \mathrm{H}$ NMR spectrum showed at 5.2-5.5 ppm for ADP-1a, ADPs1b, ADPs-2, ADPs-3a and ADPs-3b, indicating that five polysaccharides from $A$. dahuricae Radix were mainly composed of one type of sugars, which was $\alpha$ form. The chemical shifts from 3.3 to $4.5 \mathrm{ppm}$ were assigned to the $\mathrm{H}-2$ to $\mathrm{H}-6$ protons.

The ${ }^{13} \mathrm{C}$ NMR spectrum of ADP-1a and ADPs- 2 had no signal at low field from 160 to $180 \mathrm{ppm}$, which illustrated it do not contain uronic acid. The ${ }^{13} \mathrm{C}$ chemical shifts of ADP-1a and ADPs-1b was 99.51, 99.49 ppm (Fig. 5), which illustrated that it was an $\alpha$-linked residue, and it was accordance with the analysis of ${ }^{1} \mathrm{H}$ NMR. The corresponding hydrogen signal can be confirmed by the HMQC spectrum (Fig. 5) to be at 5.23, 5.24 ppm.

According to HMBC spectrum of ADP1a (Fig. 5), $\delta_{H}$ 5.23 showed correlations with the carbon signals at $\delta_{\mathrm{C}}$ 76.6, 73.2 and 71.4, $\delta_{\mathrm{H}} 3.7-3.8$ showed correlations with the carbon signals at $\delta c 76.6$ and $71.4, \delta_{\mathrm{H}} 3.53-3.67$ showed correlations with the carbon signals at $\delta c 76.6$, and $69.18, \delta_{\mathrm{H}} 3.2-3.5$ showed correlations with the carbon signals at $\delta_{\mathrm{C}} 99.5,73.2,71.4$ and 60.2. The difference between ADP1a and $\mathrm{ADP}_{\mathrm{S}}-2$ was $\delta_{\mathrm{H}} 3.53-3.67$ showed no correlations with the carbon signals.

\section{Scanning electron microscope analysis}

The SEM of ADPs-1a, ADPs-1b, ADPs-2, ADPs-3a and ADPs-3b were shown in Fig. 6. SEM images of ADPs-1a determined the surface was compact with close-packed arrays. There was multi-hole on the surface of ADPs-1b and was in flake accumulation. The surface of ADPs-2 was uneven in flocculent accumulation. The surface appearance of ADPs-3a was rough in flake accumulation. The surface topography of ADPs-3b was flat smooth in fragmental accumulation and the polysaccharide aggregate lined up tightly.

\section{Coagulation assays in vitro}

Blood coagulation is a series of enzymatic processes, including the intrinsic pathway, extrinsic pathway and internal and external common pathway, finally fibrinogen is turned into fibrin, blood is turned from the sol into a gel state. Thrombin also plays an important role in the process of coagulation and blood coagulation. Therefore, PT is used to evaluate the coagulation factors V, VII and X in the overall efficiency of extrinsic clotting pathway. APTT is a test of the coagulation factors VIII, IX, XI, XII in the intrinsic clotting activity. TT is mainly a measure of transformation of fibrinogen to fibrin degree. FIB is employed to reflect the content of fibrinogen [14].

As shown in Table 2, compared with the blank group, ADPs-1b could significantly shorten PT and TT $(P<0.001)$ and could significantly increase the content of FIB $(0.01<P<0.05)$, which indicated that ADPs-1b had procoagulant activities and exerted the procoagulant activities through intrinsic pathway, extrinsic pathway and increased the content of FIB. However, the activity of shortening PT exhibited a significant difference in relation to vitamin $\mathrm{k}_{1}(P<0.001)$. ADPs-2 could significantly shorten APTT and PT $(P<0.001)$, and both of them had significant difference with the blank group $(P<0.001)$, and thus suggested that ADPs- 2 had procoagulant activities and exerted the procoagulant activities through intrinsic pathway and extrinsic pathway. Compared with the blank group, ADPs-3a could significantly shorten APTT and TT $(0.01<P<0.05$, and $0.001<P<0.01$, respectively) and could significantly increase the content of FIB $(0.001<P<0.01)$, so the anticoagulant activities of ADPs-3a was associated with the intrinsic pathway and increased the content of FIB. Compared with the blank group, ADPs-3b could significantly shorten PT 
a

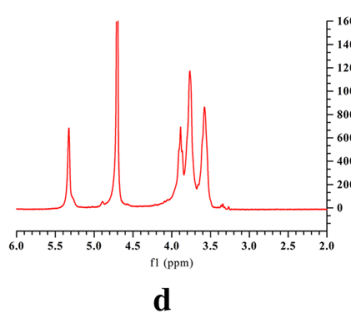

d
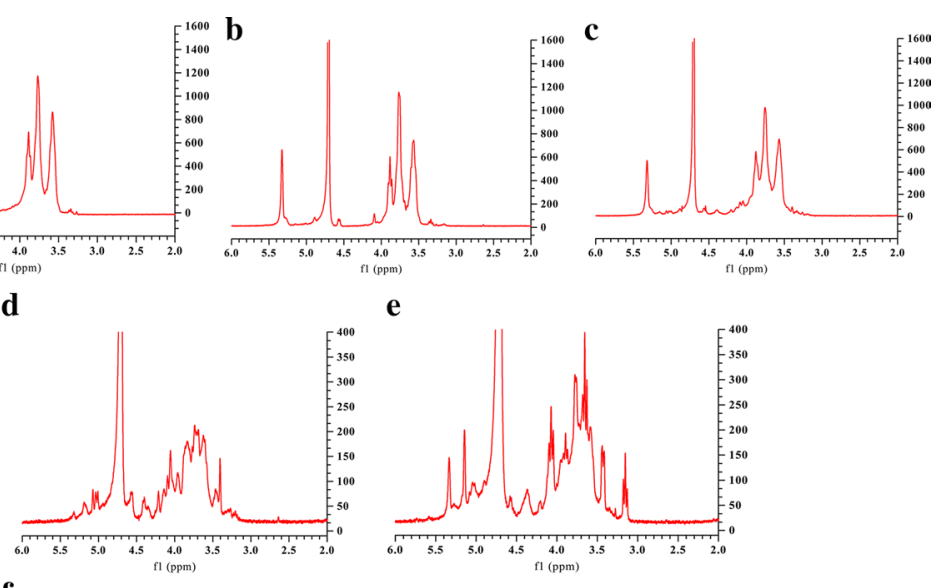

f

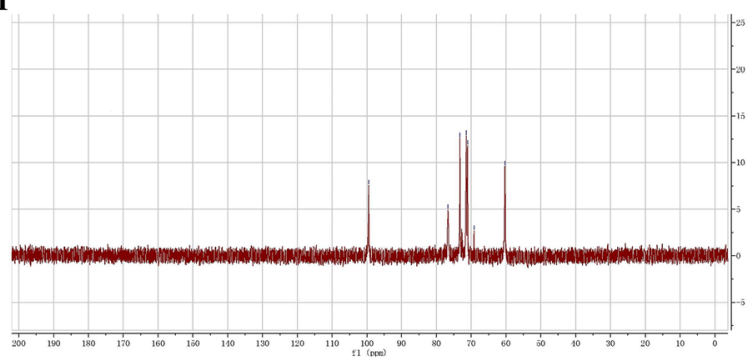

g
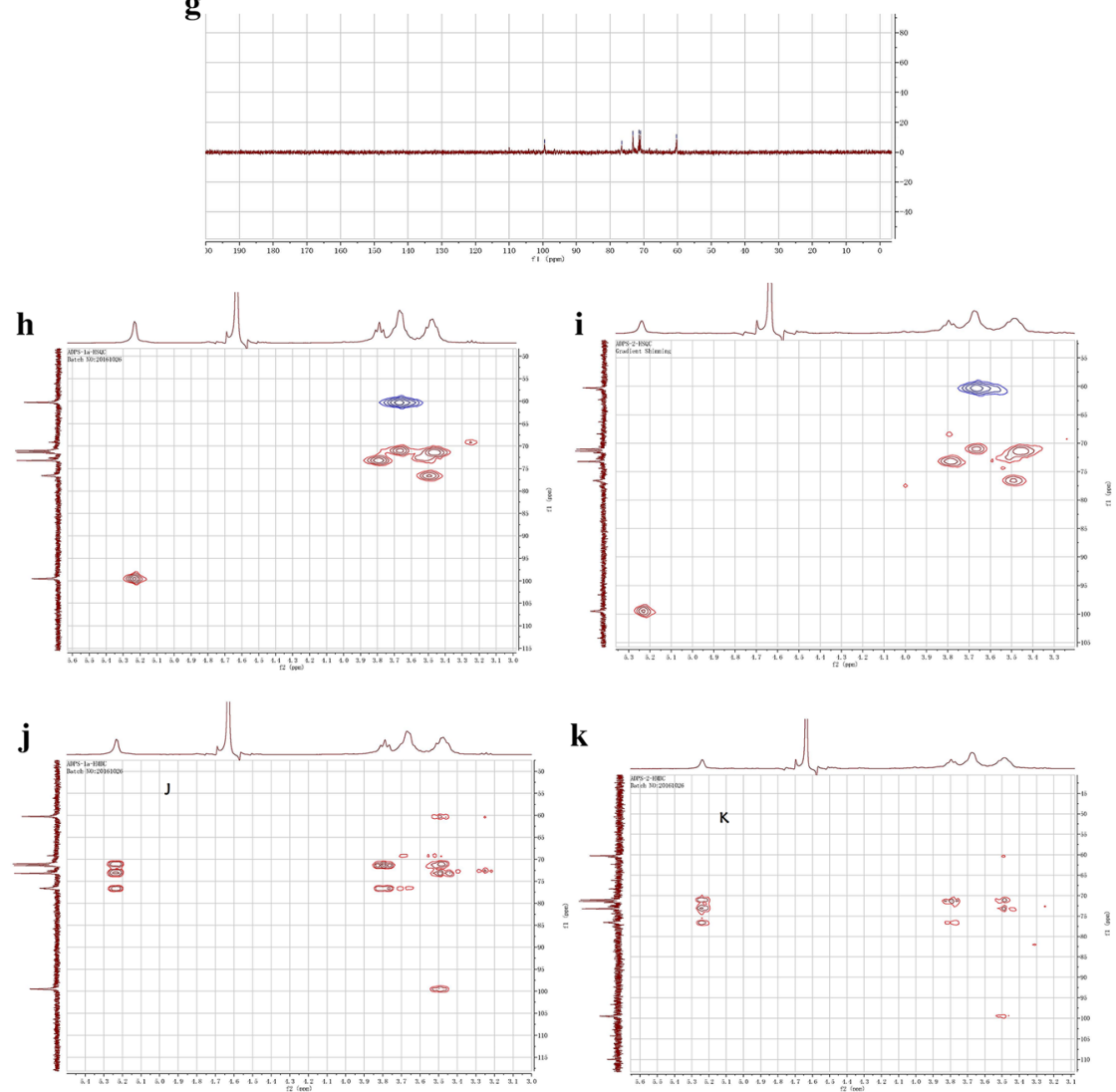

k

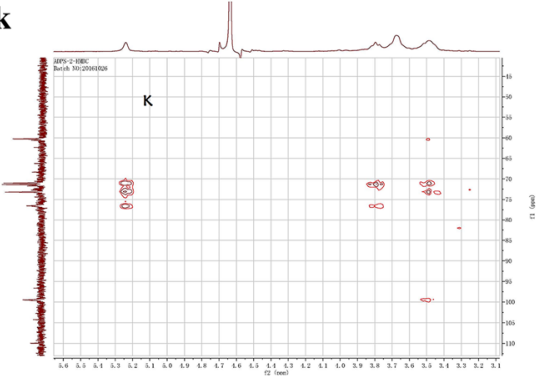

Fig. $5{ }^{1} \mathrm{H}$ NMR spectrum of ADPs-1a (a), ADPs-1b (b), ADPs-2 (c), ADPs-3a (d), ADPs-3b (e), ${ }^{13} \mathrm{C}$ NMR spectrum of ADPs-1a $(\mathbf{f}),{ }^{13} \mathrm{C}$ NMR spectrum of ADPs-2 ( $\mathbf{g})$, HMQC spectrum of ADPs-1a (h) and HMQC spectrum of ADPs-2 (i), HMBC spectrum of ADP-1a $(\mathbf{j})$ and HMBC spectrum of ADP s $^{-2}(\mathbf{k})$ 

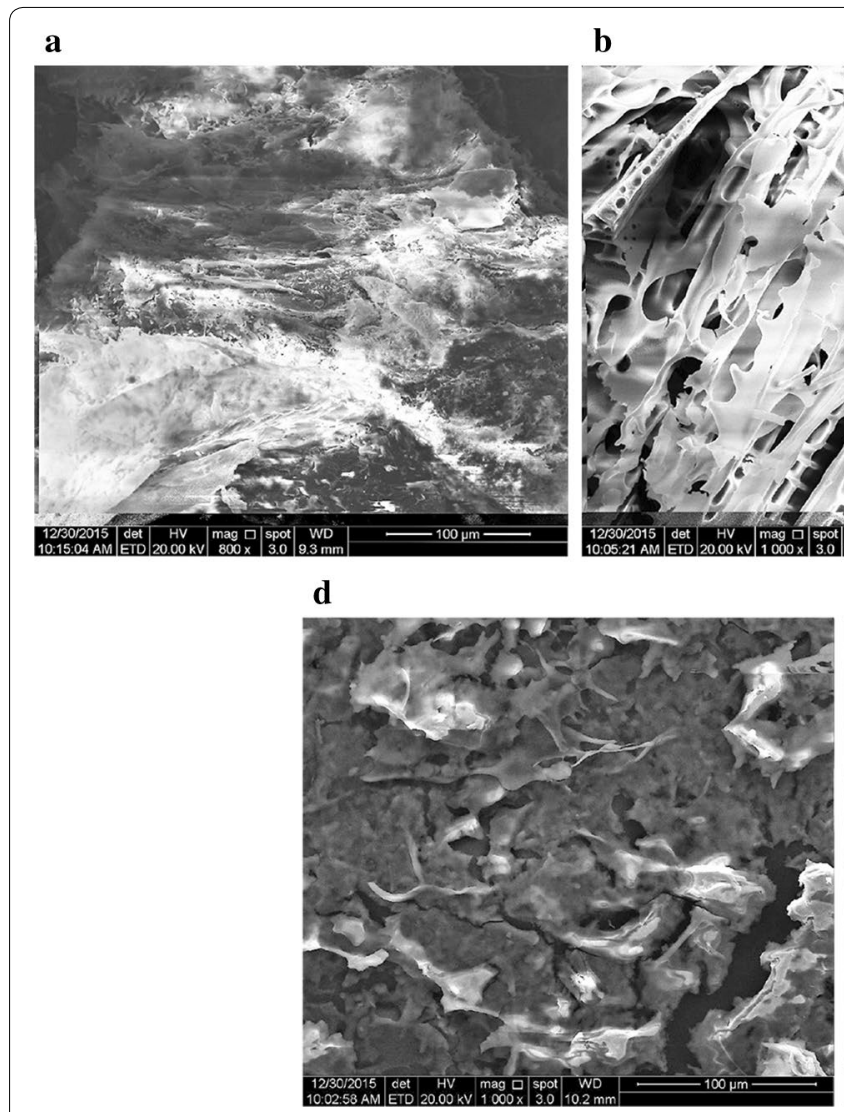

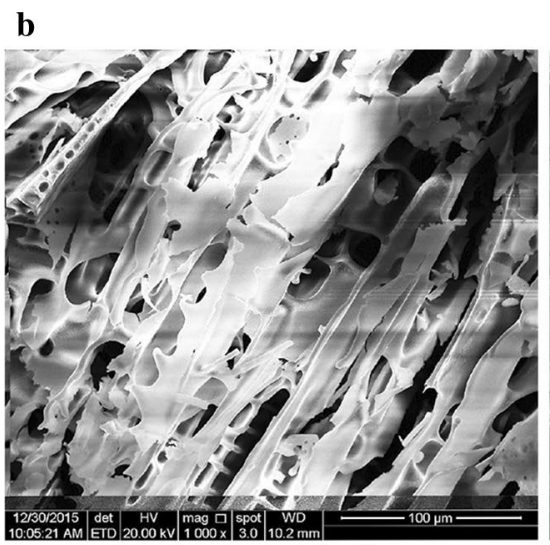

c

e

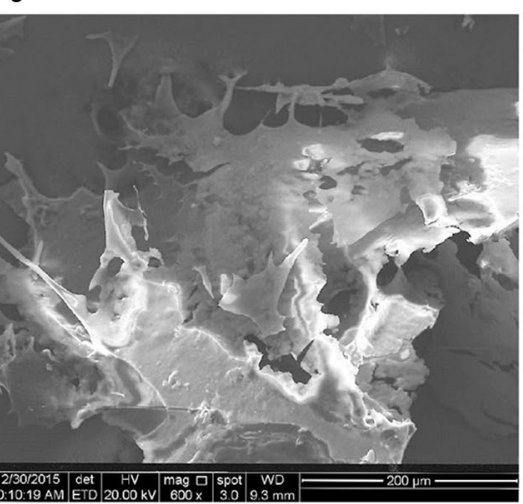

Fig. 6 SEM of ADPs-1a (a), ADPs-1b (b), ADPs-2 (c), ADPs-3a (d) and ADPs-3b (e)

Table 2 Effect of polysaccharides form Angelicae dahuricae Radix on plasma coagulation parameters

\begin{tabular}{|c|c|c|c|c|}
\hline \multirow[t]{2}{*}{ Group } & \multicolumn{4}{|c|}{ Plasma coagulation parameters } \\
\hline & APTT (s) & PT (s) & TT (s) & FIB (g/L) \\
\hline Blank & $15.23 \pm 0.32$ & $12.53 \pm 0.34$ & $15.00 \pm 0.31$ & $1.43 \pm 0.04$ \\
\hline Vitamin $\mathrm{k}_{1}$ & $14.33 \pm 0.42^{* * *}$ & $12.08 \pm 0.22^{*}$ & $13.28 \pm 0.10^{* * *}$ & $1.85 \pm 0.05^{* *}$ \\
\hline ADPs-1a & $15.27 \pm 0.31$ & $12.18 \pm 0.17$ & $14.65 \pm 0.10$ & $1.54 \pm 0.15$ \\
\hline ADPs-1b & $15.30 \pm 0.10$ & $10.50 \pm 0.26^{* * *, \&}$ & $13.60 \pm 0.24^{* * *}$ & $1.69 \pm 0.07^{*}$ \\
\hline ADPs-2 & $11.80 \pm 0.30^{* * *, \&}$ & $11.08 \pm 0.25^{* * *, \&}$ & $14.50 \pm 0.38$ & $1.63 \pm 0.20$ \\
\hline ADPs-3a & $14.73 \pm 0.12^{*}$ & $12.48 \pm 0.28$ & $14.20 \pm 0.47^{* *}$ & $2.01 \pm 0.11^{* *}$ \\
\hline ADPs-3b & $15.63 \pm 0.25$ & $11.30 \pm 0.35^{* * *, \&}$ & $14.60 \pm 0.34$ & $1.85 \pm 0.11^{*}$ \\
\hline
\end{tabular}

Data represent mean \pm SD. $n=6$

Compared with blank, ${ }^{* * *} P<0.001, * * 0.001<P<0.01, * 0.01<P<0.05$

Compared with vitamin $\mathrm{k}_{1}$, \& $P<0.001$

$(P<0.001)$, and could significantly increase the content of FIB $(0.01<P<0.05)$, which indicated that ADPs-3b had procoagulant activities and exerted the activities through extrinsic pathway and increased the content of FIB.
According to reports in the literature [12], the water soluble part from $A$. dahuricae Radix can significantly shorten the clotting time of mice. In the present study, we demonstrated that ADPs-1b, ADPs-2, ADPs-3a and ADPs-3b were the components of procoagulant activity. 


\section{Conclusions}

Five polysaccharides were extracted and purified from $A$. dahuricae Radix. Coagulation assays in vitro indicated that ADPs-1b, ADPs-2, ADPs-3a and ADPs-3b had the procoagulant activity, The results imply that polysaccharides from $A$. dahuricae Radix has promising propects as hemostatics in medicines. However, Owing to the complex relationships existed between the structure and activities of the polysaccharides, further investigations in the structure and the relationship between the fine structure and procoagulant activity are required.

\begin{abstract}
Abbreviations
HPSEC: high performance gel permeation chromatography; GC: gas chromatography; FT-IR: Fourier transform infrared; NMR: spectrometer and nuclear magnetic resonance spectra; Mw: molecular weight; APTT: activated partial thromboplastin time; PT: prothrombin time; TT: thrombin time; FIB: fibrinogen; SD: standard deviation; ANOVA: analysis of variance.
\end{abstract}

\section{Authors' contributions}

WYK and JFW conceived the research idea. PLL, JMW and QY conducted the experiments, collected the plant specimens, analyzed and interpreted the data as well as prepared the first draft. WYK, JMW, and JFW critically read and revised the paper. All authors read and approved the final manuscript.

\section{Author details \\ ${ }^{1}$ Institute of Chinese Materia Medica, Henan University, Kaifeng 475004, China. ${ }^{2}$ Kaifeng Key Laboratory of Functional Components in Health Food, Kaifeng 475004, China.}

\section{Competing interests}

The authors declare that they have no competing interests.

\section{Funding}

This work was supported by Henan Province University Science and Technology Innovation Team (16IRTSTHN019), Key project in Science and Technology Agency of Kaifeng City (1608003), Kaifeng City Science and Technology Innovation Talent (1509010), National cooperation project of Henan province (2015GH12), Key project in Science and Technology Agency of Henan Province (172102310609).
Received: 22 November 2016 Accepted: 24 January 2017

Published online: 10 February 2017

\section{References}

1. Zheng LH, Wang PJ, Li W, Feng D (2014) Chemical composition of essential oil from Angelica dahurica roots and its DPPH radical scavenging effect. Food Sci 35:180-183

2. Zhou SM (2014) Study on extraction of coumarin from Angelicae dahuricae and antimicrobial activities. Food Ind 35:141-144

3. Kleiner HE, Reed MJ, Digiovanni J (2003) Naturally occurring coumarins inhibit human cytochromes P450 and block benzo (a) pyrene and 7, 12-dimethylbenz (a) anthracene NDA adduct formation in MCF-7 cells. Chem Res Toxicol 6:415-422

4. Cui QB, Zhang Y, Lan S (2010) Study on analgesic effect of Angelica dahurica. Chin J Exp Tradit Med Formul 16:102-104

5. Sun H, Zhao QZ, Jia XD et al (2012) Study on the coumarin glucosides of Angelica dahurica. J Chin Med Mater 35(11):1785-1788

6. Li L, Lv L, Zhang W, Zhao JX, Lv DY, Lou ZY (2011) GC-MS combined with PCA analysis of the essential oil from two varieties of Radix Angelicae Dahuricae. J Pharm Anal 31:112-118

7. Li JP, Pang XH (2012) Optimization of decoloration technology for active carbon on polysaccharides from Angelicae dahuricae Radix by orthogonal test. Drugs Clin 27:366-369

8. Dai YJ, Xie CK (1990) The determination of trace elements in Angelicae Dahuricae. West China Med J 5:22-23

9. Wang DC, Gao LJ, Gao YX (2009) Antioxidative effects of polysaccharide from Radix Angelicae Dahurica in vitro. Lishizhen Med Mater Med Res 20:173-174

10. Qu JS, Kang XJ, Zheng SL (2005) Extraction of Angelica dahurica polysaccharide and its effect on rat skin cells proliferation. Chin Pharmacol Bull 21:637

11. Feng HB, Song ZH, Liu J, Zhu ZR, Du XG, Zeng XY (2014) Effects of seven Chinese herbal medicine polysaccharide on the resistance of F81 cell against parvovirus infection. Chin J Vet Med 50:51-54

12. Ou XC (1987) The observation of antithrombin effect for 126 kinds of traditional Chinese medicine. Chin Tradit Herbal Drugs 18:21

13. Kang XJ, Qu JS (2006) Analysis of Angelicae dahuricae by gas chromatography. Chin J Pharm Anal 26:891-893

14. Lou CX, Pang ZR, Zhang W, Xie K, Cui J (2009) Discussion of experiment study and mechanism of stanching in Chinese herb. J Liaoning Univ TCM 11:59-61

\section{Submit your manuscript to a SpringerOpen ${ }^{\circ}$ journal and benefit from:}

- Convenient online submission

- Rigorous peer review

- Immediate publication on acceptance

- Open access: articles freely available online

- High visibility within the field

- Retaining the copyright to your article

Submit your next manuscript at springeropen.com 